\title{
Applications of Blockchain Technology in Medicine and Healthcare: Challenges and Future Perspectives
}

\author{
Asad Ali Siyal ${ }^{1, *(0)}$, Aisha Zahid Junejo 2 (D), Muhammad Zawish ${ }^{2}$ (D) Kainat Ahmed ${ }^{3}$, \\ Aiman Khalil ${ }^{2}$ (D) and Georgia Soursou 4 \\ 1 Department of Biomedical Engineering, Mehran University of Engineering and Technology, \\ Jamshoro 76062, Pakistan \\ 2 Department of Computer Systems Engineering, Mehran University of Engineering and Technology, \\ Jamshoro 76062, Pakistan; aisha.junejo@students.muet.edu.pk (A.Z.J.); zawiish@yahoo.com (M.Z.); \\ aiman.memon32@yahoo.com (A.K.) \\ 3 School of Computer Science and Statistics, Trinity College Dublin, Dublin A94 RK10, Ireland; \\ kainatahmed80@gmail.com \\ 4 Faculty of Medicine, University of Crete, Voutes University Campus, 71202 Heraklion, Greece; \\ georgiasoursou@gmail.com \\ * Correspondence: asadalisiyal@gmail.com; Tel.: +92-333-2742374
}

Received: 6 November 2018; Accepted: 26 December 2018; Published: 2 January 2019

\begin{abstract}
Blockchain technology has gained considerable attention, with an escalating interest in a plethora of numerous applications, ranging from data management, financial services, cyber security, IoT, and food science to healthcare industry and brain research. There has been a remarkable interest witnessed in utilizing applications of blockchain for the delivery of safe and secure healthcare data management. Also, blockchain is reforming the traditional healthcare practices to a more reliable means, in terms of effective diagnosis and treatment through safe and secure data sharing. In the future, blockchain could be a technology that may potentially help in personalized, authentic, and secure healthcare by merging the entire real-time clinical data of a patient's health and presenting it in an up-to-date secure healthcare setup. In this paper, we review both the existing and latest developments in the field of healthcare by implementing blockchain as a model. We also discuss the applications of blockchain, along with the challenges faced and future perspectives.
\end{abstract}

Keywords: blockchain; healthcare, biomedical; data-sharing; medicine; distributed ledger technology; distributed systems; health information exchange, security, interoperability; transparency; privacy

\section{Introduction}

Healthcare is a data-intensive clinical domain where a huge amount of data are generated, accessed, and disseminated on a regular basis. Storing and disseminating this large amount of data is crucial, as well as significantly challenging, due to the sensitive nature of data and limiting factors, such as security and privacy [1].

In the healthcare field and clinical settings, safe, secure, and scalable (SSS) data-sharing is highly imperative for diagnosis, as well as in combined clinical decision making. The data-sharing practice is quite essential to enable clinical practitioners to transfer the clinical data of their patients to the concerned authority for a quick follow-up. Theses caregivers and general practitioners should be able to transfer the clinical data of their patients in a vastly privacy-sensitive and timely manner, to ensure that both parties have complete and up-to-date information about patient health conditions.

On the other hand, tele-medicine and e-health are two highly used domains, where clinical data is transferred remotely to a specialist (at a distant location) for an expert opinion. In these two online 
clinical setups, the patient's data is transferred either via a "store-and-forward technology", or by the means of online real-time clinical monitoring (e.g., tele-monitoring, telemetry, and the like) [2,3]. Using these online clinical settings, the patients are remotely diagnosed and treated by clinical experts by means of exchanged clinical data. In all such clinical arrangements, the security, sensitivity, and privacy of the clinical data are some of major challenges to occur, due to the case-sensitive nature of patient data. Thus, the ability to exchange data safely, securely, and scalably is highly important for supporting healthy and meaningful clinical communications, regarding remote patient cases. Because the safe and successful exchange of data helps in clinical communication by gathering recommendations or confirmations from a group of clinical specialists, which result in improved diagnostic accuracy and effective treatment [4-6].

Furthermore, there are various interoperability challenges that are faced continuously in this domain. For instance, the safe, secure, and successful exchange of clinical data between healthcare organizations or research institutions can pose severe challenges in practical operation. Such exchanges of clinical data requires substantial, trustworthy, and healthy collaboration between the entities involved. Among the potential constraints faced in this process is the nature of clinical data, sensitivity, data sharing agreements, procedures, complex patient matching algorithms, ethical policies, and governing rules. These are some of the important concerns that need to be agreed upon mutually, before carrying out any clinical data exchange practically [7].

During the past couple of years, researchers have tried to implement applications of the internet of things, artificial intelligence, machine learning, and computer vision to facilitate doctors and clinical practitioners in the diagnosis and treatment of various chronic diseases.

Recently, there has been remarkable interest in utilizing the applications of blockchains for the delivery of safe and secure healthcare data [8,9], biomedical [10] and e-health data sharing [11], brain simulation, and thinking. A blockchain follows a P2P network. It is basically a peer-to-peer integrated multi-field network framework, composed of cryptography, algorithms, and mathematical expressions aimed at solving traditional distributed database synchronization limitations by using distributed consensus algorithms. The blockchain technology principally contains six key elements: Decentralized, transparent, immutable, autonomy, open source, and anonymity (as described in Table 1).

Table 1. Key elements of blockchain technology.

\begin{tabular}{cl}
\hline Key Elements & Functionality Description \\
\hline Decentralized & $\begin{array}{l}\text { A database system with open access control to anyone connected to the network. } \\
\text { The data can be accessed, monitored, stored, and updated on multiple systems. }\end{array}$ \\
\hline Transparent & $\begin{array}{l}\text { The recorded and stored data on blockchain is transparent to potential users, } \\
\text { which can be further updated easily. The transparent nature of blockchains } \\
\text { could certainly prevent data from being altered or stolen. }\end{array}$ \\
\hline Immutable & $\begin{array}{l}\text { The records, once stored, become reserved forever and cannot be modified } \\
\text { easily without having control of more than 51\% of the node concurrently. }\end{array}$ \\
\hline \multirow{2}{*}{ Autonomy } & $\begin{array}{l}\text { The blockchain system is independent and autonomous, meaning that each } \\
\text { node on the blockchain system can access, transfer, store, and update the data } \\
\text { safely, making it trustworthy and free from any external intervention. }\end{array}$ \\
\hline Open Source & $\begin{array}{l}\text { The blockchain technology is formulated in a way that provides an open source } \\
\text { access to everyone connected to the network. This inimitable versatility entitles } \\
\text { anyone, not only to check the records publicly, but also develop various } \\
\text { impending applications. }\end{array}$ \\
\hline Anonymity & $\begin{array}{l}\text { As data transfer occurs between node to node, the identity of the individual } \\
\text { remains anonymous, thus making it a more secure and reliable system. }\end{array}$ \\
\hline
\end{tabular}

A person who is part of this network has to verify each new transaction made. As each transaction in a block of a blockchain is verified by all of the nodes in the network, it becomes more and more immutable. The diagram below shows the work flow of blockchain process (as shown in Figure 1). 


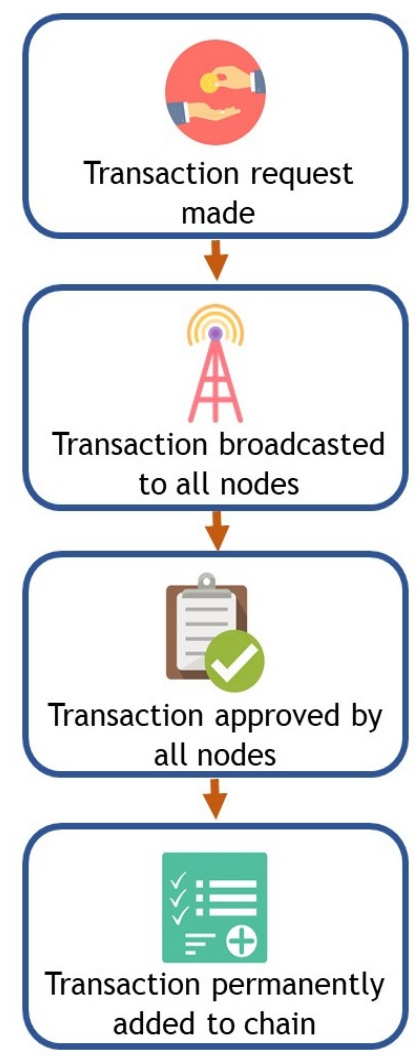

Figure 1. A generalized workflow of the blockchain process.

In future, blockchain could be a technology that may potentially help in personalized, reliable, and secure healthcare, by merging the entire real-time clinical data of a patient's health and presenting it as an up-to-date secure healthcare setup.

In this paper, we present the different forms of blockchains and discuss existing progress, as well as latest developments in the healthcare domain by implementing blockchain as a model. The rest of the paper is organized as follows: Section 2 gives a brief overview of the background information and related work done in the field of healthcare, using blockchain as a tool. Section 3 describes various applications of the emerging blockchain technology in healthcare and the medical field. Section 4 presents the challenges faced in healthcare and the medical field when utilizing blockchains. Section 5 highlights the future perspectives of the blockchain technology in the healthcare domain. The conclusion is outlined in Section 6, followed by Abbreviation and Reference sections, respectively.

\section{Related Work}

Blockchain's potential to facilitate better healthcare data-sharing, and to assist in various other diagnosis applications, has been previously described by several studies.

For instance, using a private blockchain to monitor and store personal clinical data is one of potential examples associated with the Healthcare Data Gateway (HDG) method, introduced by Yue et al. [12]. In this personalized healthcare method, the patients have the freedom to access, monitor, and manage their personal clinical data and healthcare summary, stored on a private blockchain (a centralized database system with restricted access control only entitled to authorized or specific users). In another study, Griggs et al. [13] adopted a private blockchain, based on the Ethereum protocol, to facilitate not only safe and secure use of medical sensors, and also eradicated the security risks associated with a remote patient monitoring system. Their blockchain based strategy can facilitate secure real-time remote monitoring, thus allowing practitioners to track the healthcare status of their patients from distant locations, while also maintaining a safe, secure, and up-to-date history of patients. 
Similarly, Ivan [14] demonstrated an approach towards secure health data storage by implementing a public blockchain (a decentralized database system with open access control to anyone connected to the network) for encryption. In this method, the encrypted healthcare data is stored publicly, developing a blockchain-based personal health record (PHR). Their proposed method enabled patients to have better access of their clinical data, where they can freely not only access and monitor their data, but also contribute to their record and share with any associated caregiving agency. In another study, Chen et al. [15] proposed an integrated blockchain and cloud storage-based framework, to manage and share patient's personal medical data. The proposed scheme could be used to achieve the safe and secure storage and exchange of personal patient medical data. The suggested approach is unique in its nature, as it gives patients complete access to and control over their personal medical data, thus excluding the involvement of any external third party.

Recently, Wang et al. [16] proposed a blockchain framework, based on parallel execution and artificial healthcare systems, to evaluate the healthcare status of patient diseases. The proposed method assesses the overall condition, diagnosis, and treatment process of the patient, and analyzes the associated therapeutic procedures through parallel executions and computational trials for clinical decision making. The suggested system has been tested on real, as well as artificial, healthcare systems, to evaluate the accuracy of diagnosis and effectiveness of treatment.

Jiang et al. [17] developed BloCHIE, a unique blockchain-oriented platform for healthcare information exchange. The suggested platform evaluates healthcare data sharing requirements, mainly for personal healthcare data and electronic medical records, and deals with various other forms of data by implementing blockhains within different sources. To validate the satisfactory requirements of both authenticity and privacy, they coupled the platform with on-chain and off-chain verification processes.

Adopting blockchain technology as a mechanism, medical data sharing, privacy, and security can be greatly improved between clinical specialists and healthcare entities. Following a similar mechanism, Cryan, M.A. [18] proposed a systematic and innovative architecture using blockchain technology that is capable to protect sensitive patient data, address critical data security issues, and implement a blockchain software system throughout a hospital system.

Blockchain technology has also witnessed great potential in biomedical research and clinical domains. With the practical utilization of blockchain technology, it could be possible to store all the clinical consents, plans, and protocols on a blockchain, even before commencing a clinical trail or examination. In this manner, the sensitive data related to clinical trials would be more up-to-date, secure, time stamped, and publicly transparent. On the other hand, smart contracts could also be deployed, replicated, and then executed within various phases of a clinical trial, to ensure transparency (if followed and validated correctly). In [19], Shubbar presented a blockchain-based tele-monitoring healthcare framework for the diagnosis and treatment of cancer tumors for remote patients. The suggested protocol utilizes smart contracts, along with blockchains that can be extensively used to ensure the validity and security of the patient's data at specialized medical centers, as well as in patient homes. In another study, Mannaro et al. [19] suggested DermoNet, a blockchain-based method for assisting dermatology patients via an online dermatological consultation through a tele-dermatology monitoring system. On the other hand, ProActive Aging [20] is a blockchain-based platform that helps to support the active living of aging people. It should be noted that, in extensive medical treatment processes, such as chronic diseases (e.g., cancer etc.), surgical operations, and aging, blockchain technology can be an ideal and well-suited choice. Moreover, it could be possible for pharmaceutical industries, drug manufacturers, and biomedical researchers to use DNA data stored on blockchains to conduct advanced transnational research at genomic level.

With the growing interest in information and communication technologies globally, one can foresee the future of blockchain as one of the progressing technologies of current era. According to a statistical survey, conducted in September 2015 by Statista, from 2014 onward a huge increase has been observed in the funding investment of blockchain technology worldwide (Figure 2). Thus, in the 
following years, it is quite obvious to expect blockchain technology to progress, expand and attain new avenues at a faster rate.

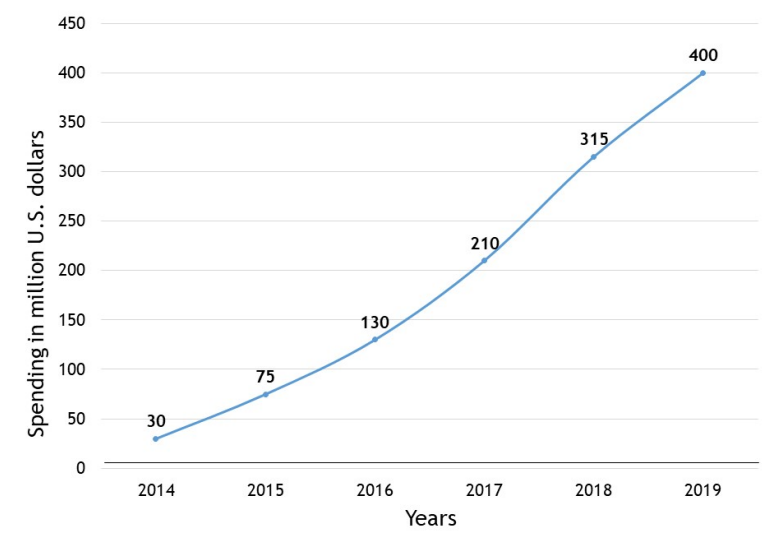

Figure 2. Capital market investments into blockchain technology worldwide, from 2014 to 2019 (in million US dollars). Survey period: 2014-15. Publication date: September 2015. Source: statista.com.

\section{Applications}

Originally, the blockchain technology (BCT) was designed for its best-known implementation in the fields of economics and cryptocurrencies, but today its utility is expanding in several other areas, including the biomedical field [10]. The potential of blockchain technology can be witnessed in the fields of medicine, genomics, telemedicine, tele-monitoring, e-health, neuroscience, and personalized healthcare applications, by its mechanism of stabilizing and securing the data set with which users can interact through different types of transactions (as depicted in the model, shown in Figure 3).

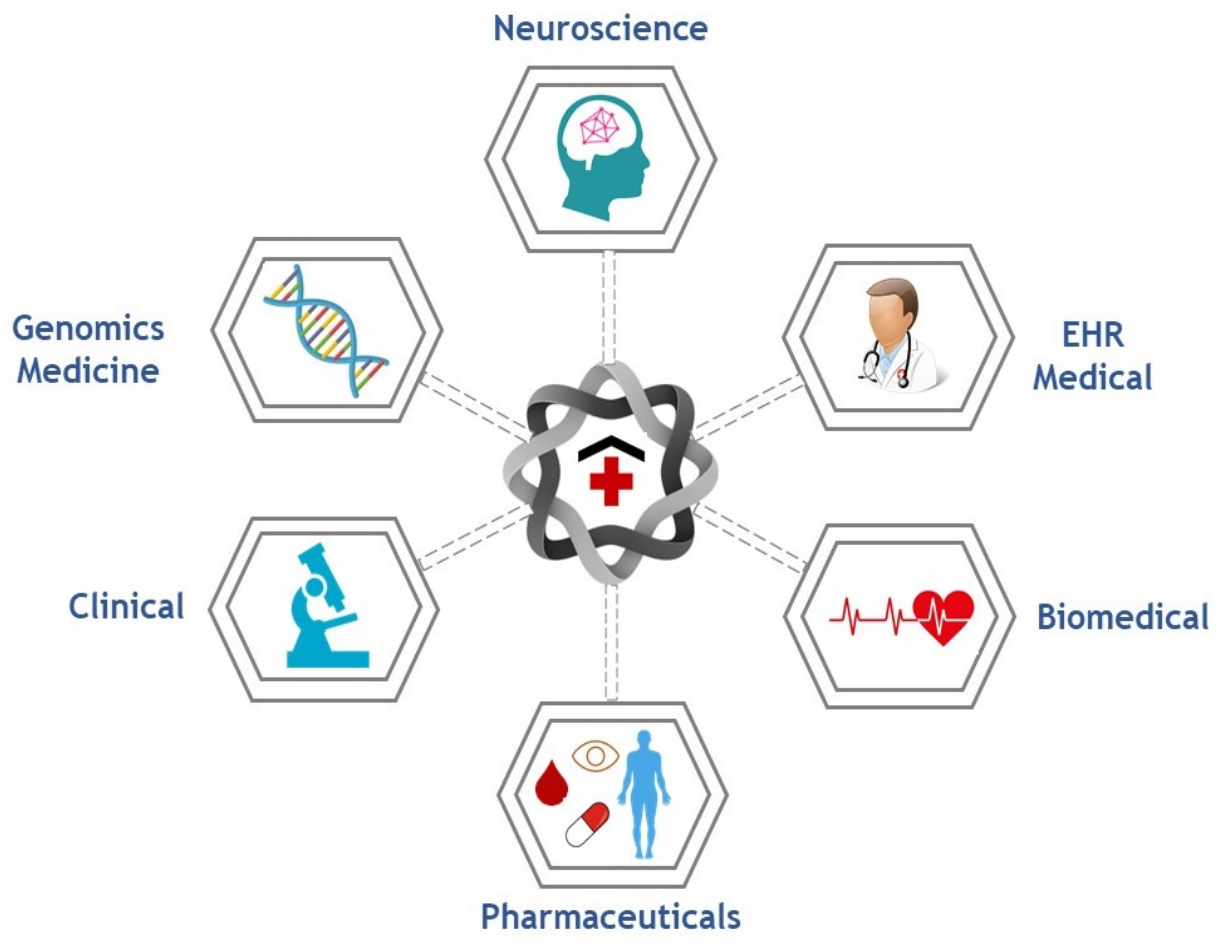

Figure 3. Applications of blockchains in healthcare. 
Some areas of healthcare and medicine, exhibiting the great potential of blockchain technology, are discussed below.

\subsection{Blockchains in Electronic Health Records (EHR)}

Over the past decade, the need of sheer increment in digitizing medical health records was caused by medical practitioners, hospitals, and healthcare devices, as digitization of this data enables easy access and sharing, and is also a basis of better and quick decision making. The most common applications of blockchain technologies in health-care are currently in the area of electronic medical records (as shown in Figure 4).

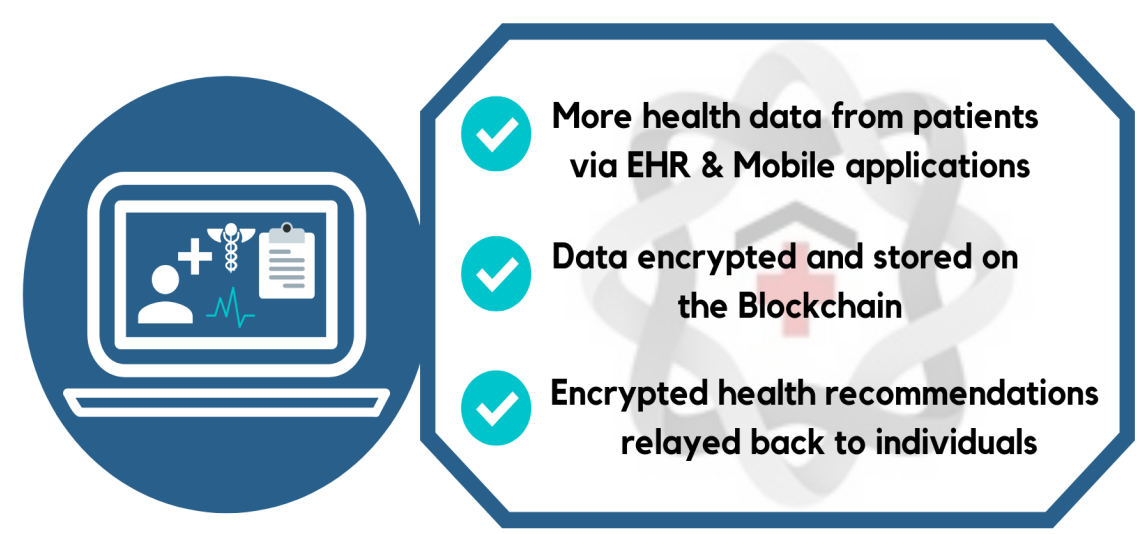

Figure 4. A depiction of interaction among EHR, mobile health applications, blockchain technology, and preventative care.

However, Electronic Health Records (EHRs) are never created to handle lifetime records among multiple institutions, and patients leave their data scattered among various institutions as life situations separate them from one provider's data into another; in this way, they lose easy access to past data [21-23]. Having faced a critical need of an innovative way to handle EHRs in a way that encourages patients to engage in their current and historical healthcare data, many researchers have brought up blockchain technology in maintaining the EHRs.

A prototype named "MedRec" utilizes distinct blockhain perks in managing authentication, confidentiality, integrity, and easy sharing of data. It works on a decentralized records management system, and claims to provide patients a detailed, immutable history and allows easy access to their respective healthcare information across various providers and treatment institutions [24].

"MedRec" doesn't store medical records or require an adjustment time. It stores a mark of the record on a blockchain and advises the patient, who is eventually responsible for where that record can move. The mark guarantees that an unchanged duplicate of the record was acquired. It likewise moves the power of control from the organization to the patient, and consequently this both weights and empowers the patient to assume the responsibility of owner. For those patients who would prefer not to deal with their information, administration associations are supposed to fill in as patient agents for this assignment. A large portion of the individual patient entrances that individuals utilize today have unwieldy plans, create more work, and have diverse UIs in each foundation. MedRec framework also includes a UI, to improve the persistent connection with healthcare records that move across multiple organizations.

During the implementation of EHR, medical data sharing often faces critical limitations, such as loss of control over data, data provenance, auditing, and secured data trailing on medical data. Keeping in view such limitations, Xia et al. [25] introduced MeDShare, a safe and secured blockchain system for medical data exchange among untrusted parties. MeDShare could be used to share medical data and maintain electronic health records among cloud service providers, hospitals, and healthcare research 
entities, with greater data provenance, personalized audit control, and minimal possible threats to data security and privacy.

EHR generally contain highly-sensitive and critical data related to patients, which is often frequently shared among clinicians, radiologists, healthcare providers, pharmacists, and researchers, for effective diagnosis and treatment. During storage, transmission, and distribution of this highly-sensitive patient information among several entities, the patient's treatment can be compromised, which can pose severe threats to patient health and in maintaining the up-to-date patient history. In the case of patients fighting with chronic diseases (e.g., cancer and HIV), the prevalence of such risks can become higher due to a long history of pre-and-post treatment, follow-ups, and rehabilitation processes. Thus, maintaining an up-to-date patient history has become highly imperative, to ensure effective treatment. To circumvent such limitations, Dubovitskaya et al. [26] proposed a blockchain-based framework for managing, maintaining, and sharing the electronic medical records of cancer patients. They adopted a permissioned blockchain technology to access, manage, and store encrypted patient data. Such proposed frameworks can be used to practically implement blockchain technology for accessing and managing the privacy and security of patient data and history in clinical practices.

Another benchmark in history is the Estonian medical record blockchain-based project. In 2016, Estonia emerged with global leadership in blockchain technology, when it proposed the idea of keeping millions of medical records private, and simultaneously making them widely available to medical providers and insurance companies [27].

Perhaps the reason of global growth of using blockchain technology in medicine is a strong assurance to patients, in using this technology to make his/her healthcare records immutable and unaltered. Any effort of access or adjustment can be quickly labeled and recognized all throughout the blockchain. This isn't only useful for patient integrity, but also identifies any criminal exercises, including wholesale fraud or adulteration of records. Moreover, approved medicinal service record sharing and review will be significantly simpler. At the point when a patient visit happens, it tends to be seen by the majority of that patient's suppliers quickly. Through suitable patient-caring algorithms, medication bugs, hypersensitivities, and drug solutions can be accommodated over all blockchain records fairly quickly, without the requirement for tedious pharmaceutical compromise forms. The utilization of blockchain innovation will accordingly encourage improved access to care, medical record management [28], prompt clinical information confirmation, expanded security, and more effective arrangement of care.

\subsection{Blockchains in Clinical Research}

A range of issues including data privacy, data integrity, data sharing, record keeping, patient enrolling [29], and so on, may arise in clinical trials. Blockchain, being the next internet generation [30], can provide viable solutions to these problems.

Healthcare researchers are working on resolving these issues with the help of blockchain technology [31,32]. The healthcare industry will soon be taken by storm with the applications of blockchain, accompanied by artificial intelligence (AI) and machine learning.

In the study proposed by Timothy et al. [31], permissioned Ethereum, a protocol that provides smart contract functionality in blockchain [32,33], is used parallel to clinic based data management systems. The main focus of the study was to address the issue of the patient enrolment problem. The findings of the study showed that Ethereum resulted in quicker transactions, as compared to bitcoin, and hence the derived conclusion proposed the use of Ethereum smart contracts for transparency of data management systems in clinical trials. Therefore, patient enrolment using blockchains is one of the existing applications of this technology in clinical research.

Another research was conducted by Mehdi Benchoufi [34]: A framework was implemented to take the informed consent of patients for tracking and storing it in such a way that it is secure, publicly verifiable, and unfalsifiable. They used a blockchain technology to create its workflow. 


\subsection{Blockchains in Medical Fraud Detection}

One huge application of blockchains in medical industry includes medicinal drug supply chain management. Supply management is a crucial issue to safeguard in all sectors, but it has a greater importance in healthcare, due to its increasing complexity. This is because any compromise to the healthcare supply chain effects the wellbeing of a patient [35]. Supply chains are vulnerable, and consists of holes for fraudulent attacks as they involve a number of moving parts and people. Blockchains provide a safe and secure platform to eliminate this problem and, in some cases, prevent fraud occurrence as well, by introducing higher data transparency and improved product traceability. Since a record in blockchain can only be validated and updated through a smart contract, manipulating the blockchain isn't easy [36].

\subsection{Blockchains in Neuroscience}

The amount of news and analysis devoted to blockchain applications is booming, and the neuroscience discipline is certainly included [37]. Modern neural technologies seek to shape a new paradigm that excludes mechanical interaction with the surrounding infrastructure, and allows one to control devices and data through mental commands. Such neural devices can interpret the patterns of brain activity and translate them into commands for controlling external devices, as well as detecting the current mental state of a person, based on data of their brain activity. The special task of reading and interpreting brain signals is solved by neural interface devices equipped with several sensitive sensors, computing chips, and wireless communication. They read the electrical activity of the brain, which is further deciphered and transmitted to the equipment under control. All of this happens in one device, which the person wears on their head. Compex algorithms and big data will utilize blockchain philosophy to store those brain signals on the neural interface. One of the companies to confirm that they'll be using blockchain tech is Neurogress. Registered in Geneva and formed in 2017, the firm is focused on building neural-control systems, enabling users to control robotic arms, drones, smart appliances, and AR/VR (augmented reality/virtual reality) devices with their own thoughts.

Neurogress' control system is based around using machine learning to improve its brain-reading accuracy, something which requires retaining $90 \%$ of brain-data, in order to train the AI being used by the system. In other words, the "big data of user neural activity" is demanded, with the company's whitepaper citing the Human Brain Project's need for "exabytes ( 1 exabyte $=1$ billion of gigabytes) of memory", as an example of the kind of storage capacity necessary. It's therefore unsurprising that Neurogress plans to use blockchain, which it believes "effectively addresses the problem of data storage security and privacy". By recording user data on a decentralised blockchain, this data becomes "resistant to hacking attacks" and thereby more private. At the same time, use of blockchain technology makes the Neurogress system "open and transparent to potential users of the Neurogress platform services". Because any abnormal activity would be easily traceable, the system will "ensure security and confidentiality of personal data".

It is therefore evident that blockchains are a form of information technology with several important future applications, capable of supporting brain augmentation, simulation of the brain, and brain thinking. Digitising an entire human brain obviously requires some medium in which to store it, and it's here that blockchain technology rears its head once again. One proposal is the storing of mindfiles, which would act as building blocks of data in personal thinking chains, shareable in a peer-to-peer network file system that allows historical versioning. This type of blockchain thinking is proposed as an input-processing-output computational system, with several features that lend opportunity to artificial intelligence, human enhancement, and their potential integration. Blockchain allows an interconnected network of computers to shake hands at intervals of a timestamp to validate the source and truth of a ledger. If we were to build a brain from the ground up, this type of trust mechanism could enable nets of neurons to store and recall information with accuracy and trust of what is subjective versus objective of a given experience. The multi-factor authentication connecting to a personal thinking chain, as a blockchain implementation, can enable opportunity for the safe 
building of a quantified-self data commons for human beings. Such a data commons reduces the siloes of human data, while also allowing each human to keep ownership over the privacy or sharing of their experience, possibly to garner monetary benefit without the use of a third party or centralised authority. At some future date, using an augmented version of this technology, when two or more people experience the same moment, though from subjective perspectives, we could reassemble their experiences to be more objective about the happenings of that moment. Ideally, this would enable the creation of virtual simulations for past memories, and the potential to see subjectively from someone else's perspective. Once we have a better elastic understanding of individual mappings to emotions and sensory experiences as contributive to a given memory, this would take in data from the senses onto this future blockchain (i.e., sight, smell, and so forth.). And the fact is, the technologies to make this a reality are being developed. In the not-so-distant future, we can begin recording our sensory experiences by use of wearable technology, the current state of brain and nerve implants, biofeedback imaging, and any other sensors that permit a multi-factor fingerprint specific to a given human's record of temporal experience. Using these technologies as a starting point, research can be performed in the improvement of decision making, learning, recall, and rehabilitative protocols.

\subsection{Blockchains in Pharmaceutical Industry and Research}

The pharmaceutical industry is one of the largest-growing, and is a leading sector at the forefront of healthcare delivery. The pharmaceutical sector not only helps in the introduction of new and potential drugs into the market, but also assists in ensuring the safety and validity of medical products and drugs sold to the end consumer. Besides, the pharmaceutical sector also aids in evaluation [28] and processing of safe drugs, which assist ultimately in quicker patient recovery. In the usual cases, drug companies face the challenges of tracking their products timely, which sometimes leads to pose severe risks by allowing counterfeiters to compromise the production, or invade fake drugs into the system. Consequently, the production and distribution of counterfeit drugs has become one of the major and global health risks, particularly in developing countries. During the production, as well as research and development (R\&D), of these drugs, blockchain could be a best-fit technology, which can be used for evaluating, monitoring, and ensuring the production processes of potential drugs. Recently, Hyperledger [38], a research foundation, launched a counterfeit medicine project using blockchain technology as a foremost tool, for inspecting and fighting the production of counterfeit drugs. When it comes to effective delivery of reliable and authentic drugs to patients, there is a dire need to monitor, evaluate, and ensure the overall process of developing and supplying pharmaceutical drugs through the use of digital technologies worldwide, and particularly in developing countries. In this regard, a digital drug control system (DDCS) [39] could be a durable solution towards the prevention of counterfeit drugs. Using a blockchain-based DDCS, big pharmaceutical industries (Sanofi, Pfizer, and Amgen) launched a joint pilot project for inspection and the evaluation of new drugs [40]. Using blockchain as an approach, it would be possible to not only track the production and location of the drugs at any given time, but also to improve the traceablitiy of falsified drugs [41], security of the drug supply system [42], and guarantee the quality of drugs supplied to consumers or end-users [39].

The applications of adopting blockchain to advance biomedical/healthcare research are summarized in Table 2. 
Table 2. Applications of blockchain technology in healthcare.

\begin{tabular}{cl}
\hline Applications & Summary \\
\hline $\begin{array}{c}\text { Electronic } \\
\text { Health } \\
\text { Records }\end{array}$ & $\begin{array}{l}\text { A digital EHR on a distributed ledger of an allowed blockchain is guaranteed } \\
\text { with the integrity, from the stage of data generation to the point of data retrieval, } \\
\text { without human intercession. }\end{array}$ \\
\hline $\begin{array}{c}\text { Clinical } \\
\text { Research }\end{array}$ & $\begin{array}{l}\text { Blockchain introduces a decentralized secure framework for any information } \\
\text { collaborations that could happen, with regards to clinical research. With this, } \\
\text { data can be securely shared with groups of researchers. }\end{array}$ \\
\hline $\begin{array}{c}\text { Medical } \\
\text { Fraud }\end{array}$ & $\begin{array}{l}\text { Blockchain, having the feature of being immutable, helps in fraud detection by } \\
\text { not allowing any duplication or modification in the transaction, and eventually } \\
\text { allows a transparent and secure transaction. }\end{array}$ \\
\hline $\begin{array}{c}\text { Neuroscience } \\
\text { Research }\end{array}$ & $\begin{array}{l}\text { Blockchain, as an innovation, brings several upcoming applications } \\
\text { incorporating brain augmentation, reenactment of the brain, and brain thinking. } \\
\text { Digitizing a whole human brain clearly requires some medium in which to } \\
\text { store it, and it's here that blockchain innovation raises its head. }\end{array}$ \\
\hline $\begin{array}{c}\text { Pharmaceutical } \\
\text { Industry and } \\
\text { Research }\end{array}$ & $\begin{array}{l}\text { Blockchain, using its power of detailed tracing, keeps eye on every stage of the } \\
\text { pharmaceutical supply chain: The origin of the medicine, its components, and } \\
\text { ownership are frequently detected at each stage to avoid the forging/stealing } \\
\text { of goods. }\end{array}$ \\
\hline
\end{tabular}

\section{Challenges}

Blockchain is an emerging technology, spreading in various sectors [43] with a high amount of benefits [44] and opportunities [45]. However, this technology comes with its own set of challenges that should be addressed (as depicted in Figure 5). A few of these major challenges are discussed in this section.

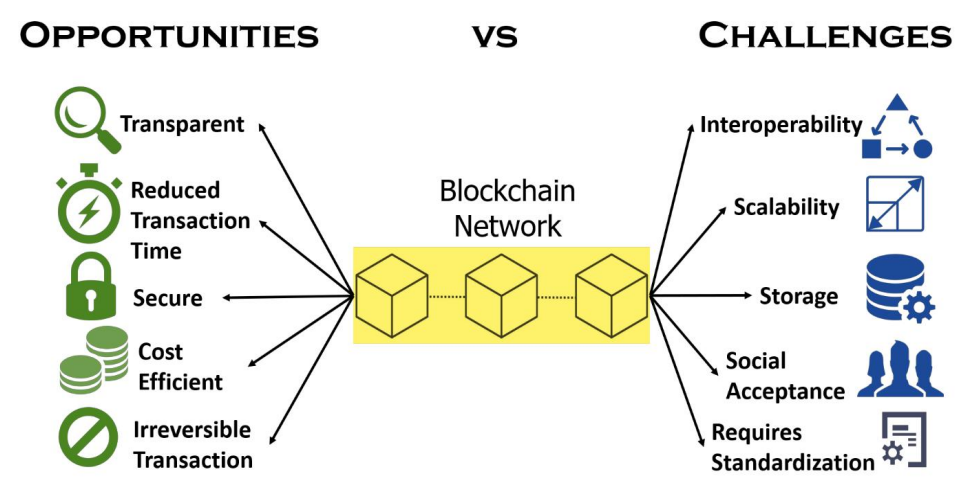

Figure 5. Opportunities and challenges of blockhains in healthcare.

\subsection{Security and Privacy of Data}

The very first, and the most crucial, challenge is the security and privacy of data [46]. With implementation of applications based on the technology of blockchain, the need of a third party to carry out a transaction is eliminated [44]. Since the mechanism of blockchain allows the entire community, rather than a single trusted third party, to verify the records in a blockchain architecture [47], the data becomes prone to potential privacy and security risks. Since all nodes can access the data transmitted by one node, the data privacy will not be intact. Absence of a third party for authorization requires the patient to select one or more representatives that can access his information and/or medical history on their behalf, in the case of an emergency. Now, this representative can further allow a set of people to access the records of the same patient, which may create a huge data privacy and security threat. Involving high-security mechanisms to the data will, in turn, result in hurdles in transferring the data from one block to another and, thus, the receivers will have access to limited or incomplete data. Further, blockchain networks are prone to a kind of security breach 
known as $51 \%$ attack [48]. This attack involves a team of miners that own more than $50 \%$ of the blocks in a blockchain network. The miners get an authority of the network and could prevent any new transactions taking place by not providing them with the consent. According to coindesk [49], five cryptocurrencies have been a victim of this attack recently. Also, a patient record might have sensitive data that is unsuitable to be on the blockchain [50].

\subsection{Managing Storage Capacity}

Another challenge that appears on this front is management of storage capacity. Blockchain was designed to record and process the transaction data, which has a limited scope, so it doesn't need heavy storage [51]. With time, as it spread its wings into the domain of healthcare, storage challenges became evident. The healthcare sector has a large amount of data that must be processed on a daily basis. From patient records, health history, and test reports, to MRI Scans, X-rays, and other medical images-all the data, in the blockchain scenario, will be available to all the nodes in the chain, which requires a massive storage space [52,53]. Moreover, applications of blockchain are transaction-based, and hence the databases used for this technology have a tendency to grow at a rapid rate. Due to increasing size of databases, the speed of record-searching and -accessing becomes low, which is highly unsuitable for the types of transactions where speed is essential. Therefore, a blockchain solution needs to be scalable and resilient [54].

\subsection{Interoperability Issues}

Blockchain also suffers from the issue of interoperability [46]; that is, making blockchains from various communicating providers and services talk to one another seamlessly and appropriately. This challenge creates hindrances in the effective sharing of data [55].

\subsection{Standardization Challenges}

Blockchain technology is still in its infancy, and thus towards its practical implementation in medicine and healthcare it will certainly face standardization challenges. A number of well-authenticated and certified standards would be required from international standardization authorities. These predefined standards would be helpful to evaluate the size, data nature, and format of the information exchanged in blockchain applications. These standards will not only scrutinize the shared data, but also must serve as precautionary safety measures.

\subsection{Social Challenges}

Blockchain technology is still evolving, and therefore faces social challenges, like cultural shift, besides aforementioned technical challenges. Accepting and adopting a technology that is completely different from the traditional work methods never comes easy. Although the medical industry is slowly moving towards digitization, there's still a long way to go for it to completely move on to this technology, especially to ones like blockchain—which has yet not been validated in clinical aspects. Convincing doctors to switch from paperwork to making use of technology will take time and effort. Due to its low adoption rate in the health sector, the technology and policies offered are relatively untrusted [54]. Due to all of these challenges and threats, we cannot, to date, term it as a viable and universal solution for all issues in healthcare [44].

In order to further understand, examine, and identify the strengths, weaknesses, opportunities, and threats faced by blockchain technology in the healthcare domain, we have carried out a SWOT analysis approach (as depicted in Figure 6). 


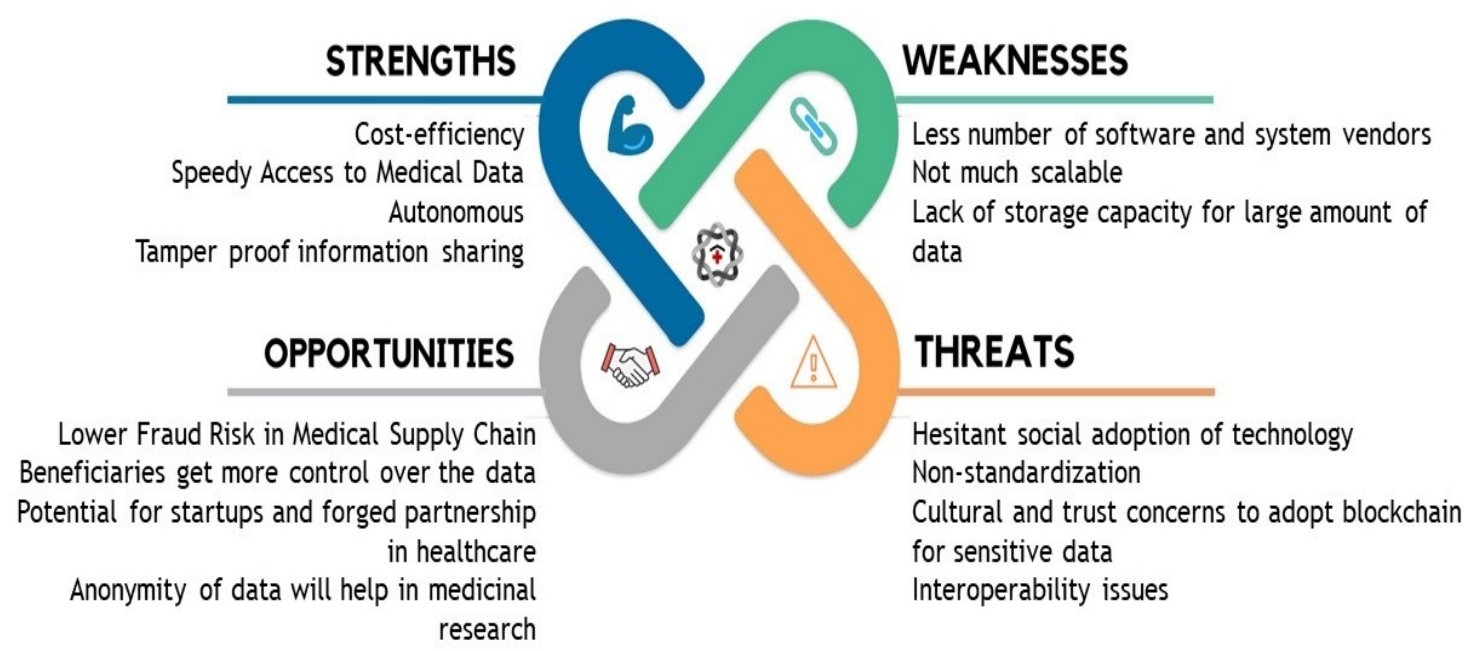

Figure 6. SWOT analysis for blockchains in healthcare.

\section{Future Perspectives}

Blockchain technology has numerous advantages to offer to the medical industry. The way the internet revolutionized healthcare and introduced telemedicine, similarly, the blockchain technology is likely to take medical science to the next level in the future, by reducing the costs of monitoring, configuration, and having a central server for data, and for the administration managing the medical data. Employing blockchains in clinical contexts will drastically reduce processing time, because as soon as a patient enrolls in a study, complete collection of data will be available at once, due to availability on the distributed ledger.

Moreover, doctors won't have to worry about the patient giving them an honest medical history, due to their ability to view the original, authentic, and quality source-documented data in real time; reducing any probable medical history errors. Likewise, the patients won't have to worry about having a second opinion from another doctor, due to the transparency of the data. Having patient records on a blockchain network will lead to people knowing and connecting with numerous others, all around the world, with similar medical conditions as they possess, which will not only be beneficial for their health, but will also result in the patients feeling accepted, supported, and to have strengthened willpower to fight the disease. Patients will have a complete autonomy on their data, and they will decide who to share the data with. This coming era can be termed as Freedom-As-A-Service, as proposed by Richie Etwaru at a book launch in 2016.

\section{Implications and Conclusions}

In spite of the fact that healthcare, once in a while, lingers behind different ventures in receiving new advancements, due to the integrity of the potential data, there may be motivation to grasp blockchain in healthcare in the near future. Managing electronic health records in the blockchain could have a few focal points. The discussed application of smart contracts encourages us to adopt them, to take care of every minor and significant exchange in the healthcare industry. Smart contracts would give an unbreakable chain of blocks, that would take into consideration individualized care without breaking into clinical frameworks. Converging clinical systems, through a blockchain smart contract, would resist the production of any duplicate copy in the parent centralized system. A potential assistance of blockchain for researchers is that it could provide verified and timestamped versions of scientific studies. Just like the smart contracts allow patients to control their data, a documentation blockchain record would allow researchers to have a sustainable history of their findings. In the giant field of the pharmaceutical industry, blockchain is a must-have technology. 
With blocking, it is conceivable to decipher all report dissemination of pharmaceutical associations into a blockchain. This arrangement will expand the speed of preparing data, guarantee straightforwardness of archive dissemination, and reduce the likelihood of loss, harm, or fabrication of archives. These points of interest are controlled by the innovation itself: The made block can't be transformed, it can't be erased. Blockchain will guarantee that data can't be adulterated. The offer of substandard or fake drugs will be rejected. This is accomplished through the control of all components in the chain of generation, logistics, and sale of medicines.

The practical utilization of blockchain technology in the healthcare domain will benefit a large number of individuals, medical practitioners, healthcare providers, R\&D specialists, healthcare entities, and biomedical researchers to effectively disseminate the vast amount of data, share clinical knowledge, and communicate recommendations with greater security and guaranteed privacy protection. The successful implementation of blockchain technology in healthcare clinical settings would surely open new research avenues for the advancement of biomedical research. While, on the other hand, in precision medicine applications, the safe, secure, and scalable acquisition, storage, and sharing of this clinical data would assist in developing potential strategies for the diagnosis and treatment of diseases. A blockchain could be used for neural-control systems, and a digitized brain could be stored on a blockchain. Neurotechnology remains at a very experimental stage, and only a select few companies have gone so far as confirming a role for blockchain technology.

The question remains, however, as to just how safe personal brain data would remain on a blockchain. The decentralised and transparent nature of blockchains would certainly prevent data from being altered or stolen, but many of the general concerns regarding large-scale data collection still apply: That sensitive data might end up being sold to third parties for questionable marketing purposes, and that users might still be indirectly identifiable (as they are with bitcoin) via pseudonymous identifiers or patterns of data.

Consequently, this blockchain-based healthcare framework will engage individuals more in their healthcare, which will ultimately improve the quality of life in a more befitting manner.

Author Contributions: All authors contributed equally to this work. All authors wrote, reviewed, and commented on the manuscript. All authors have read and approved the final version of the manuscript.

Funding: This research received no external funding.

Conflicts of Interest: The authors declare no conflict of interest.

\section{Abbreviations}

The following abbreviations are used in this manuscript:

SSS Safe, secure and scalable

HDG Healthcare data gateway

PHR Personal health record

EHR Electronic health record

AI Artificial intelligence

R\&D Research and development

BCT Bockchain technology

DDSC Digital drug control system

\section{References}

1. Griebel, L.; Prokosch, H.U.; Köpcke, F.; Toddenroth, D.; Christoph, J.; Leb, I.; Engel, I.; Sedlmayr, M. A scoping review of cloud computing in healthcare. BMC Med. Inform. Decis. Mak. 2015, 15, 17. [CrossRef] [PubMed]

2. Houston, M.S.; Myers, J.D.; Levens, S.P.; McEvoy, M.T.; Smith, S.A.; Khandheria, B.K.; Shen, W.K.; Torchia, M.E.; Berry, D.J. Clinical consultations using store-and-forward telemedicine technology. In Mayo Clinic Proceedings; Elsevier: Rochester, MN, USA, 1999; Volume 74. 
3. Bhatti, A.; Siyal, A.A.; Mehdi, A.; Shah, H.; Kumar, H.; Bohyo, M.A. Development of cost-effective tele-monitoring system for remote area patients. In Proceedings of the 2018 IEEE International Conference on Engineering and Emerging Technologies (ICEET), Lahore, Pakistan, 22-23 February 2018.

4. Castaneda, C.; Nalley, K.; Mannion, C.; Bhattacharyya, P.; Blake, P.; Pecora, A.; Goy, A.; Suh, K.S. Clinical decision support systems for improving diagnostic accuracy and achieving precision medicine. J. Clin. Bioinform. 2015, 5, 4. [CrossRef] [PubMed]

5. Zhang, P.; White, J.; Schmidt, D.C.; Lenz, G.; Rosenbloom, S.T. Fhirchain: Applying blockchain to securely and scalably share clinical data. Comput. Struct. Biotechnol. J. 2018, 16, 267-278. [CrossRef] [PubMed]

6. Berman, M.; Fenaughty, A. Technology and managed care: Patient benefits of telemedicine in a rural health care network. Health Econ. 2005, 14, 559-573. [CrossRef] [PubMed]

7. Downing, N.L.; Adler-Milstein, J.; Palma, J.P.; Lane, S.; Eisenberg, M.; Sharp, C.; Longhurst, C.A. Health information exchange policies of 11 diverse health systems and the associated impact on volume of exchange. J. Am. Med. Inform. Assoc. 2017, 24, 113-122. [CrossRef] [PubMed]

8. Azaria, A.; Ekblaw, A.; Vieira, T.; Lippman, A. MedRec: Using Blockchain for Medical Data Access and Permission Management. In Proceedings of the 2nd International Conference on Open and Big Data (OBD 16), Vienna, Austria, 22-24 August 2016; pp. 25-30.

9. Zhang, J.; Xue, N.; Huang, X. A Secure System for Pervasive Social NetworkBased Healthcare. IEEE Access 2016, 4, 9239-9250. [CrossRef]

10. Kuo, T.-T.; Kim, H.; Ohno-Machado, L. Blockchain distributed ledger technologies for biomedical and health care applications. J. Am. Med. Inform. Assoc. 2017, 24, 1211-1220. [CrossRef] [PubMed]

11. Angraal, S.; Krumholz, H.M.; Schulz, W.L. Blockchain technology: Applications in health care. Circ. Cardiovasc. Qual. Outcomes 2017, 10, e003800. [CrossRef] [PubMed]

12. Yue, X.; Wang, H.; Jin, D.; Li, M.; Jiang, W. Healthcare data gateways: Found healthcare intelligence on Blockchain with novel privacy risk control. J. Med. Syst. 2016, 40, 218. [CrossRef] [PubMed]

13. Griggs, K.N.; Ossipova, O.; Kohlios, C.P.; Baccarini, A.N.; Howson, E.A.; Hayajneh, T. Healthcare Blockchain System Using Smart Contracts for Secure Automated Remote Patient Monitoring. J. Med. Syst. 2018, 42, 130. [CrossRef]

14. Ivan, D. Moving toward a blockchain-based method for the secure storage of patient records. In ONC/NIST Use of Blockchain for Healthcare and Research Workshop; ONC/NIST: Gaithersburg, MD, USA, 2016.

15. Chen, Y.; Ding, S.; Xu, Z.; Zheng, H.; Yang, S. Blockchain-Based Medical Records Secure Storage and Medical Service Framework. J. Med. Syst. 2018, 43, 5. [CrossRef] [PubMed]

16. Wang, S.; Wang, J.; Wang, X.; Qiu, T.; Yuan, Y.; Ouyang, L.; Guo, Y.; Wang, F.Y. Blockchain-Powered Parallel Healthcare Systems Based on the ACP Approach. IEEE Trans. Comput. Soc. Syst. 2018, 99, 1-9. [CrossRef]

17. Jiang, S.; Cao, J.; Wu, H.; Yang, Y.; Ma, M.; He, J. Blochie: A blockchain-based platform for healthcare information exchange. In Proceedings of the 2018 IEEE International Conference on Smart Computing (SMARTCOMP), Taormina, Italy, 18-20 June 2018.

18. Cyran, M.A. Blockchain as a Foundation for Sharing Healthcare Data. Blockchain in Healthcare Today. Blockchain Healthc. Today 2018. [CrossRef]

19. Shubbar, S. Ultrasound Medical Imaging Systems Using Telemedicine and Blockchain for Remote Monitoring of Responses to Neoadjuvant Chemotherapy in Women's Breast Cancer: Concept and Implementation. Master's Thesis, Kent State University, Kent, OH, USA, 2017.

20. Ianculescu, M.; Stanciu, A.; Bica, O.; Neagu, G. Innovative, Adapted Online Services that Can Support the Active, Healthy and Independent Living of Ageing People. A Case Study. Int. J. Econ. Manag. Syst. 2017, 2, 321-329.

21. Mandl, K.D.; Markwell, D.; MacDonald, R.; Szolovits, P.; Kohane, I.S. Public Standards and Patients' Control: how to keep electronic medical records accessible but private. BMJ 2001, 322, 283-287. [CrossRef] [PubMed]

22. Brandon, R.M.; Podhorzer, M.; Pollak, T.H. Premiums without benefits: Waste and inefficiency in the commercial health insurance industry. Int. J. Health Serv. 1991, 21, 265-283. [CrossRef] [PubMed]

23. Gorman, L. The history of health care costs and health insurance. Wisconsin Policy Res. Inst. Rep. 2006, 19, $1-31$.

24. Ekblaw, A.; Azaria, A.; Halamka, J.D.; Lippman, A. A Case Study for Blockchain in Healthcare: "MedRec" prototype for electronic health records and medical research data. In Proceedings of the IEEE Open and Big Data Conference, Vienna, Austria, 24 August 2016; Volume 13. 
25. Xia, Q.; Sifah, E.B.; Asamoah, K.O.; Gao, J.; Du, X.; Guizani, M. MeDShare: Trust-less medical data sharing among cloud service providers via blockchain. IEEE Access 2017, 5, 14757-14767. [CrossRef]

26. Dubovitskaya, A.; Xu, Z.; Ryu, S.; Schumacher, M.; Wang, F. Secure and trustable electronic medical records sharing using blockchain. In AMIA Annual Symposium Proceedings; American Medical Informatics Association: Washington, DC, USA, 2017; Volume 2017, p. 650.

27. Heston, T. A case study in blockchain health care innovation. Int. J. Curr. Res. 2017, 9, 60587-60588. [CrossRef]

28. Mettler, M. Blockchain technology in healthcare: The revolution starts here. In Proceedings of the 2016 IEEE 18th International Conference on e-Health Networking, Applications and Services (Healthcom), Munich, Germany, 14-16 September 2016.

29. How Blockchain Will Revolutionise Clinical Trials, June 2018. Available online: https:/ / pharmaphorum. com/views-and-analysis/how-blockchain-will-revolutionise-clinical-trials-clinical-trials / (accessed on 20 November 2018).

30. Moe Alsumidaie, Blockchain Concepts Emerge in Clinical Trials, Applied Clinical Trials, May 2018. Available online: http:/ / www.appliedclinicaltrialsonline.com/blockchain-concepts-emerge-clinical-trials (accessed on 15 October 2018).

31. Nugent, T.; Upton, D.; Cimpoesu, M. Improving data transparency in clinical trials using blockchain smart contracts. F1000Research 2016, 5, 2541. [CrossRef]

32. Buterin V: A Next-Generation Smart Contract And Decentralized Application Platform; White Paper; Ethereum Foundation (Stiftung Ethereum): Zug, Switzerland, 2014.

33. Wood G: Ethereum: A Secure Decentralised Generalised Transaction Ledger; Yellow Paper; Ethereum Foundation (Stiftung Ethereum): Zug, Switzerland, 2014.

34. Benchoufi, M.; Porcher, R.; Ravaud, P. Blockchain protocols in clinical trials: Transparency and traceability of consent. F1000Research 2017, 6, 66. [CrossRef]

35. Kevin. A.C.; Breeden, E.A.; Davidson, C.; Mackey, T.K. Leveraging Blockchain Technology to Enhance Supply Chain Management in Healthcare: An exploration of challenges and opportunities in the health supply chain. Blockchain Healthc. Today 2018. [CrossRef]

36. Mauri, R. Blockchain for Fraud Prevention: Industry Use Cases. July 2017. Available online: https://www. ibm.com/blogs/blockchain/2017/07/blockchain-for-fraud-prevention-industry-use-cases / (accessed on 16 October 2018).

37. Swan, M. Blockchain thinking: The brain as a decentralized autonomous corporation. IEEE Technol. Soc. Mag. 2015, 34, 41-52. [CrossRef]

38. Taylor, P. Applying Blockchain Technology to Medicine Traceability; April 2016. Available online: https: / www.securingindustry.com/pharmaceuticals/applying-blockchain-technology-to-medicinetraceability/s40/a2766/ (accessed on 16 October 2018).

39. Plotnikov, Vladimir, and Valentina Kuznetsova. The Prospects for the Use of Digital Technology "Blockchain" in the Pharmaceutical Market. In MATEC Web of Conferences; EDP Sciences: London, UK, 2018; Volume 193.

40. Markov, A. Use of Blockchain in Pharmaceutics and Medicine. Available online: https:/ / miningbitcoinguide. com/technology / blokchejn-v-meditsine/ (accessed on 16 October 2018).

41. Sylim, P.; Liu, F.; Marcelo, A.; Fontelo, P. Blockchain Technology for Detecting Falsified and Substandard Drugs in Distribution: Pharmaceutical Supply Chain Intervention. JMIR Res. Protoc. 2018, 7, e10163. [CrossRef] [PubMed]

42. Trujllo, G.; Guillermo, C. The Role of Blockchain in the Pharmaceutical Industry Supply Chain as a Tool for Reducing the Flow of Counterfeit Drugs. Ph.D. Thesis, Dublin Business School, Dublin, Ireland, 2018.

43. Shae, Z.; Tsai, J.J. On the design of a blockchain platform for clinical trial and precision medicine. In Proceedings of the 2017 IEEE 37th International Conference on Distributed Computing Systems (ICDCS), Atlanta, GA, USA, 5-8 June 2017.

44. Alhadhrami, Z.; Alghfeli, S.; Alghfeli, M.; Abedlla, J.A.; Shuaib, K. Introducing blockchains for healthcare. In Proceedings of the 2017 IEEE International Conference on Electrical and Computing Technologies and Applications (ICECTA), Ras Al Khaimah, UAE, 21-23 November 2017.

45. Fernández-Caramés, T.M.; Fraga-Lamas, P. A Review on the Use of Blockchain for the Internet of Things. IEEE Access 2018, 6, 32979-33001. [CrossRef] 
46. Kuo, T.-T.; Hsu, C.-N.; Ohno-Machado, L. ModelChain: Decentralized Privacy-Preserving Healthcare Predictive Modeling Framework on Private Blockchain Networks. arXiv 2016, arXiv:1802.01746 .

47. Zheng, Z.; Xie, S.; Dai, H.; Chen, X.; Wang, H. An overview of blockchain technology: Architecture, consensus, and future trends. In Proceedings of the 2017 IEEE International Congress on Big Data (BigData Congress), Honolulu, HI, USA, 25-30 June 2017.

48. Investopedia "Blockchains". Available online: https://www.investopedia.com/terms/1/51-attack.asp (accessed on 20 October 2018).

49. Hertig, A. Blockchain's Once-Feared 51 Percent Attack Is Now Becoming Regular. June 2018. Available online: https: / www.coindesk.com/blockchains-feared-51-attack-now-becoming-regular/ (accessed on 20 October 2018).

50. Linn, L.A., Koo, M.B. Blockchain for health data and its potential use in health it and health care related research. In ONC/NIST Use of Blockchain for Healthcare and Research Workshop; ONC/NIST: Gaithersburg, MD, USA, 2016.

51. Esposito, C.; De Santis, A.; Tortora, G.; Chang, H.; Choo, K.K. Blockchain: A Panacea for Healthcare Cloud-Based Data Security and Privacy? IEEE Cloud Comput. 2018, 5, 31-37. [CrossRef]

52. Bennett, B. Blockchain HIE Overview: A Framework for Healthcare Interoperability. Telehealth Med. Today 2017, 2. [CrossRef]

53. Pirtle, C.; Ehrenfeld, J. Blockchain for Healthcare: The Next Generation of Medical Records? J. Med. Syst. 2018, 42, 172. [CrossRef] [PubMed]

54. McKinlay, J. Blockchain: Background Challenges and Legal Issues; DLA Piper Publications: London, UK, 2016.

55. Boulos, M.N.; Wilson, J.T.; Clauson, K.A. Geospatial blockchain: Promises, challenges, and scenarios in health and healthcare. Int. J. Health Geogr. 2018, 25. [CrossRef]

(C) 2019 by the authors. Licensee MDPI, Basel, Switzerland. This article is an open access article distributed under the terms and conditions of the Creative Commons Attribution (CC BY) license (http://creativecommons.org/licenses/by/4.0/). 